\title{
ECHOCARDIOGRAPHIC EVALUATION OF THE FUNCTIONAL MITRAL REGURGITATION AFTER CARDIAC RESYNCHRONIZATION THERAPY: WHICH IS THE BEST APPROACH?
}

\author{
Despina M. TOADER ${ }^{1 凶}$, Constantin BATAIOSU ${ }^{2}$, Rodica MUSETESCU ${ }^{3}$ \\ ${ }^{1}$ Craiova Cardiology Center - EuroEchoLab, Craiova, Romania \\ ${ }^{2}$ Craiova Cardiology Center - Electrophysiology Department, Craiova, Romania \\ ${ }^{3}$ University of Medicine and Pharmacy Craiova, Faculty of Medicine, Cardiology Department, Craiova, \\ Romania
}

Received 02 June 2020, Accepted 05 Aug 2020
hitps://doi.org/10.31688/ABMU.2020.55.3.13

\begin{abstract}
A strong association between functional mitral regurgitation severity and all-cause mortality and hospitalization was found in patients with dilated cardiomyopathy and left ventricular systolic dysfunction, with or without an implanted device. Many studies revealed a bidirectional link between the decrease in functional mitral regurgitation severity and the response to cardiac resynchronization therapy. This emphasizes the importance to find methods for mitral regurgitation evaluation in heart failure patients after device implantation. In this complex pathology, adequate measurement remains a challenge for clinicians. Echocardiography is the key technique used to confirm the diagnosis and to assess the severity and prognosis. In this review, we discuss the echocardiographic challenges in the evaluation of functional mitral regurgitation after cardiac resynchronization therapy. An integrated approach including various criteria is strongly recommended. The assessment includes the integration of data from 2D/3D echocardiography imaging, as well as Doppler measures of severity. For quantifying the regurgitation
\end{abstract}

\section{RÉsumé}

Évaluation échocardiographique de la régurgitation mitrale fonctionnelle après la thérapie de resynchronisation cardiaque : quelle est la meilleure approche?

Une forte association entre la gravité de la régurgitation mitrale fonctionnelle et la mortalité de toute cause et l'hospitalisation a été observée chez les patients atteints de cardiomyopathie dilatée et de dysfonction systolique ventriculaire gauche, avec ou sans dispositif implanté. De nombreuses études ont révélé un lien bidirectionnel entre la diminution de la gravité de la régurgitation mitrale fonctionnelle et la réponse à la thérapie de resynchronisation cardiaque. Cela souligne l'importance de trouver des méthodes d'évaluation de la régurgitation mitrale chez les patients atteints d'insuffisance cardiaque après l'implantation du dispositif. Dans cette pathologie complexe, une mesure adéquate reste un défi pour les cliniciens. L'échocardiographie est la technique clé utilisée pour confirmer le diagnostic et évaluer la gravité et le pronostic. Dans cette revue, nous discutons des défis échocardiographiques 
degree, the vena contracta width and the parameters derived from the PISA method, effective regurgitant orifice area and regurgitant volume, are the most useful. There is also important to provide information about the left ventricle and right ventricle dimensions and ejection fraction, as well as the left atrium, right atrium dimensions and the pulmonary arterial systolic pressure.

Keywords: functional mitral regurgitation, echocardiography, cardiac resynchronization therapy.

\section{List of abbreviations: \\ CRT: cardiac resynchronization therapy \\ DCM: dilated cardiomyopathy \\ FMR: functional mitral regurgitation \\ LVEF: left ventricle ejection fraction \\ PISA: proximal isovelocity surface area \\ VC: vena contracta}

\section{INTRODUCTION}

The estimated prevalence of functional mitral regurgitation (FMR) in dilated cardiomyopathy (DCM) ranges from $8 \%$ to $74 \%$ in echocardiography studies, approximately $50 \%$ in ischemic cardiomyopathy and $56-65 \%$ in nonischemic cardiomyopathy. ${ }^{1}$ A strong association between moderate to severe FMR and all-cause mortality and hospitalization was found in patients with DCM and left ventricular systolic dysfunction, with or without an implanted device. ${ }^{1}$ Many studies revealed that a decrease in FMR severity is a determinant of the response to cardiac resynchronization therapy (CRT). ${ }^{2}$ This emphasizes the importance to find methods for FMR evaluation after CRT. An adequate assessment of FMR mechanisms and severity remains a challenge for clinicians.

In this review, we discuss the echocardiographic challenges in the evaluation of FMR after CRT.

\section{Mechanisms of FMR}

FMR is a complex pathology and occurs in valves without organic lesions. The main determinant is "systolic tenting", due to displacement of the leaflets coaptation point away from mitral annulus towards the left ventricle cavity. ${ }^{3}$ FMR contributes in turn to worsening of the left ventricular remodeling, creating a vicious circle in which left ventricular dilatation and FMR act as self-perpetuating processes. ${ }^{3}$ There are many factors involved in FMR development in patients with DCM, but the key is represented by an imbalance between the closing forces and the tethering forces: dans l'évaluation de la régurgitation mitrale fonctionnelle après thérapie de resynchronisation cardiaque. Une approche intégrée comprenant divers critères est fortement recommandée. L'évaluation comprend l'intégration des données de l'imagerie d'échocardiographie 2D / 3D ainsi que des mesures de gravité Doppler. Pour quantifier le degré de régurgitation, la largeur de la vena contracta et les paramètres dérivés de la méthode PISA: la surface effective de l'orifice régurgitant et le volume régurgitant sont les plus utiles. Il est également important de fournir des informations sur les dimensions et la fraction d'éjection du ventricule gauche et du ventricule droit ainsi que sur les dimensions d'oreillette gauche, d'oreillette droite et la pression systolique artérielle pulmonaire.

Mots-clés: régurgitation mitrale fonctionnelle, échocardiographie, thérapie de resynchronisation cardiaque.

- The main determinant of the decreased closing force is left ventricular contractile dysfunction.

- The main determinant of the increased tethering force is the displacement of papillary muscles ${ }^{4}$

The components involved in the appearance and evolution of FMR are:

1. Left ventricular remodeling: which may be local (after an acute myocardial infarction) or global (DCM, volume or pressure overload) ${ }^{4}$.

2. Mitral apparatus. Depending on global or local left ventricular remodeling, two phenotypes of tenting have been described:

- the asymmetric pattern, characterized by posterior displacement of the posterior leaflet, the papillary muscles and the coaptation point. This pattern is more frequent in inferolateral myocardial infarctions. The jet direction is opposite to the affected leaflet and it is related to a lesser degree, to left ventricular dysfunction. ${ }^{3}$

- the symmetric pattern, in which both leaflets are similarly affected due to the apical displacement of both papillary muscles. In this case, the coaptation point is set apically and the regurgitant jet is central. This group of patients presents a more advanced stage of left ventricular dilatation and dysfunction. ${ }^{3}$

3. Mitral annulus dilation/dysfunction reduces mitral valve coaptation and worsens FMR. In patients with FMR, the mitral annulus has a larger size and a flattened shape. The studies showed that isolated annulus dilation does not lead to FMR in the absence of mitral leaflet tethering. ${ }^{5}$ Annular dilation/dysfunction was considered 
a factor which predisposes to increase of mitral valve tenting area. ${ }^{5}$

4. Cardiac dyssynchrony:

- Atrioventricular dyssynchrony is involved in late diastolic regurgitation. ${ }^{4}$

- Interventricular dyssynchrony between the left ventricle and the right ventricle affects mainly the interventricular septal motion and its contribution to left ventricular ejection. ${ }^{4}$

- Intraventricular dyssynchrony refers to differences in the timing of contraction between myocardial segments of the left ventricle (LV), reducing the systolic performance. ${ }^{5}$

Several studies have shown that the dyssynchrony of myocardium surrounding the papillary muscles is also related to the development of FMR., ${ }^{5,13}$

Depending on hemodynamic parameters, FMR usually presents intra-beat variability, due to phasic changes of regurgitant volume, greater in early and late systoles, and lower in the mid-systole. Beat-to-beat variation may also be found. ${ }^{6}$

\section{FMR IN PATIENTS WITH CRT}

According to latest European Society of Cardiology (ESC) guideline for the diagnosis and treatment of acute and chronic heart failure, CRT to improve symptoms and reduce morbidity and mortality is indicated in symptomatic patients in sinus rhythm with a QRS duration of 130-149 msec and left bundle branch block QRS morphology and with left ventricle ejection fraction (LVEF) $<35 \%$ despite optimal medical treatment (Class I, level B) and it should be considered for symptomatic patients with HF in sinus rhythm with a QRS duration $\geq 150 \mathrm{msec}$ and non-left bundle branch block QRS morphology and with LVEF $<35 \%$ despite optimal medical treatment (Class IIa, level B). ${ }^{1}$

The prevalence of FMR after CRT varies in different studies. FMR may diminish, remain stable, or worsen after implantation and has been identified as a prognostic factor in patients under CRT. ${ }^{2} \mathrm{~A}$ reduction of mitral regurgitation severity of at least one degree was observed in $30-40 \%$ of patients after implant, independently of the etiology of the underlying cardiomyopathy. It was found an association between FMR and mortality, independent of left ventricular volume changes and clinical CRT response. ${ }^{2}$ Many studies demonstrated that a decrease in FMR severity is a determinant of response to CRT, and the responders had a poorer outcome if the regurgitation persisted. ${ }^{2}$

\section{The LinK Between FMR and CRT}

Echocardiographic evaluation of patients with implanted devices demonstrated that CRT may correct the pathophysiologic determinants of FMR. The benefit derives from the correction of left ventricular dyssynchrony and reverse remodeling. ${ }^{4}$

The specific mechanisms of CRT on FMR are:

1. Restoration of the left ventricle and papillary muscles synchronous contraction improves spatial relations and function of the sub valvular apparatus and the mitral valve (decreasing in tethering force).

2. Improved coordination of left ventricular wall contraction contributes to an increase in trans mitral pressure gradient (increasing in closing force). ${ }^{4}$

Other mechanisms involved in FMR reduction in patients with CRT are:

3. Reverse remodeling of the left ventricle at the later phase of CRT.

4. Mitral valve annular size and function correction.

5. Optimization of atrioventricular delay generates an adequate trans mitral pressure gradient and eliminates diastolic mitral regurgitation. ${ }^{4}$

The response of FMR to CRT has two phases:

- Immediate, short-term FMR reduction occurring directly after the implantation, that is the major determinant of a favorable response. The tethering forces are diminished by a better left ventricular contraction, including papillary muscles-bearing segments, and closing forces are increased by improvement in the trans mitral pressure gradient. Due to the correction of the atrioventricular delay, diastolic or pre-systolic mitral regurgitation is eliminated. ${ }^{4}$

- Long-term FMR reduction occurring after weeks to months, as a consequence of reverse remodeling. In this phase, the tethering forces are reduced due to a decrease in left ventricular volume and sphericity, and closing forces are increased due to the improvement of left ventricular systolic function. ${ }^{4}$

\section{FMR ECHOCARDIOGRAPHIC EVALUATION IN PA- TIENTS WITH CRT}

Echocardiography is the key technique used to confirm the diagnosis of FMR and to assess its severity and prognosis. An integrated approach including various criteria is strongly recommended. ${ }^{3}$

The guidelines recommend two-dimensional transthoracic echocardiography as first-line imaging in valvular regurgitation and this is often sufficient for diagnosis. This approach is also applied in FMR after CRT? Two-dimensional transesophageal echocardiography and three-dimensional 
echocardiography provide supplementary data when the transthoracic evaluation is of non-diagnostic value or in complex valve lesions.

Transesophageal echocardiography permits obtaining information about underlying anatomical conditions and FMR mechanism, but due to changes in left ventricular loading conditions during the transesophageal evaluation, the severity of regurgitation may be underestimated. This favors transthoracic echocardiography as a method of choice for FMR quantification. $^{?}$

\section{D TTE ECHOCARDIOGRAPHY FMR QUANTIFICA- TION}

The qualitative evaluation includes:

- The mitral valve morphology, which must be visually assessed in multiple views. The mitral annulus must be evaluated using the parasternal long-axis view. Dilatation is diagnosed when the ratio annulus/anterior leaflet is $>1.3$ in diastole or when the annulus diameter is $>35 \mathrm{~mm}^{7}$ (Figure 1, panel A). The normal contraction of the mitral annulus is evaluated as a decrease in the annular area in systole with $25 \%$. In this view, tenting area and tenting height (Figure 1, panel B) can also be measured.? From the parasternal short-axis view, the interpapillary muscle distance is obtained in end-systole ${ }^{7}$ (Figure 1, panel C).

- Continuous wave Doppler of mitral regurgitation jet is a qualitative complementary parameter of severity (Figure 1, panel D). A dense signal indicates severe mitral regurgitation, as well as a truncated (notched) envelope, with a triangular contour or an early peak velocity (blunt). Diastolic FMR can be evaluated $^{7}$ (Figure 1, panel E).

- Color flow Doppler regurgitant jet is the most common way to assess mitral regurgitation presence. This parameter evaluates the spatial orientation of the jet and could be used just to differentiate between and mild vs severe regurgitation and for localization of the origin of the regurgitant jet. Qualitatively, the presence of flow convergence at a Nyquist limit of $50-60 \mathrm{~cm} / \mathrm{s}$ is an indicator of severe mitral regurgitation. ${ }^{6}$

Theoretically, larger color jets that extend deep into the let atrium represent more severe mitral regurgitation than smaller jets. According to the guidelines, a large central jet, or eccentric, adhering, swirling and reaching the posterior wall of the left atrium is a marker of severe mitral regurgitation. ${ }^{6}$

The relationship between jet size and mitral regurgitation severity is influenced by many technical factors: transducer frequency, gain, Nyquist limit, frame rate, and hemodynamic factors: the eccentricity of the jet, and flow momentum, which is the product of flow rate and velocity. ${ }^{7}$ This method underestimates eccentric jets (Coanda effect) ${ }^{7}$.

The guidelines recommend using the color flow imaging only for detecting mitral regurgitation. A more quantitative approach is needed for more than a small central mitral regurgitation jet.

Semiquantitative parameters:

- Vena contracta (VC) width. This is the smallest width of the jet, immediately distal to the regurgitant orifice, perpendicular to the direction of the jet and reflects the regurgitant orifice area. ${ }^{6}$ It is measured in a view perpendicular to the commissural line. Averaging measurements over at least two to three beats and using two orthogonal planes are recommended?

In secondary mitral regurgitation, the regurgitant orifice is elongated along the mitral coaptation line. In consequence, $\mathrm{VC}$ is narrow in the four-chamber view (Figure 2, Panel A) and broad (Figure 2, Panel B) in the two-chamber view. An average of VC widths (four- and two-chamber views) was better correlated with the 3D VC area and a value $>8 \mathrm{~mm}$ on 2D echo has been reported to define severe FMR. ${ }^{6}$

This method is quick and easy to use, relatively independent of hemodynamic and instrumentation factors and not affected by another valve leak. It can be used for differentiating between mild vs. severe regurgitation and also for an eccentric jet, but it is affected by systolic changes in regurgitant flow.? In the case of multiple jets, in 2D transthoracic examination, the values of the $\mathrm{VC}$ width are not additive.

$\mathrm{VC}$ is one of the parameters recommended by guidelines to quantify mitral regurgitation. ${ }^{?}$

Quantitative parameters are obtained from the flow convergence method (proximal iso velocity surface area - PISA method), which estimates the effective regurgitant orifice area and regurgitation volume. ${ }^{3}$ Effective regurgitant orifice area $>20 \mathrm{~mm}^{2}$ and regurgitation volume $>30 \mathrm{~mL}$ are indicators of severe FMR and identify a subset of patients at an increased risk of cardiovascular events ${ }^{6}$ (Figure 2, Panel C).

Dynamic variation of the regurgitant orifice area with early and late systolic peaks and a mid-systolic decrease can be better observed using the color M-mode (Figure 2, Panel D). These changes reflect the phasic variation in trans mitral pressure that acts to close the mitral leaflets. ${ }^{7}$ The PISA method is founded on the assumption of hemispheric symmetry of the velocity distribution proximal to the circular regurgitant lesion. This may be affected by the aliasing velocity, non-circular orifice, systolic changes in regurgitant flow, and adjacent structures.? In FMR, the PISA has an ellipsoidal shape and may be observed two separate mitral regurgitation jets 


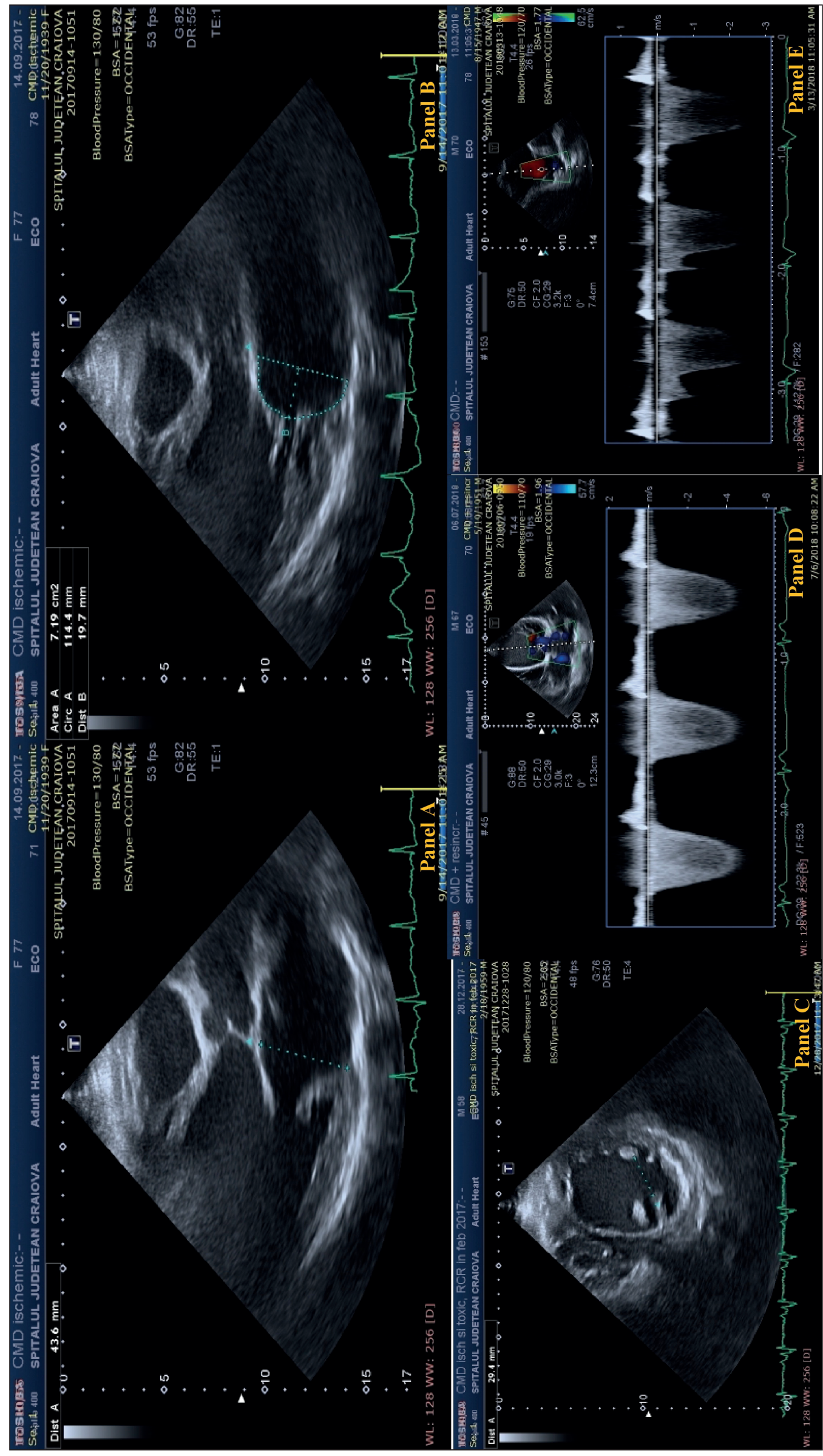

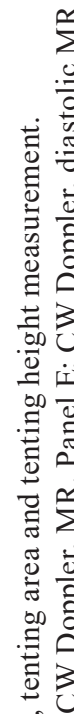

鄫

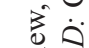

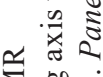

$\sum \stackrel{\text { bo }}{=}$

岁要

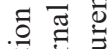

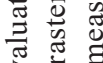

ठ च

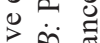

可

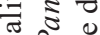

$2 \stackrel{0}{0}$

$\dot{-\dot{0}} \vec{g}$

ํㅣㄹ

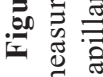

묨

蒮.苛

志

要茬

$\forall$ 물

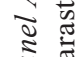

ह

ปั) 


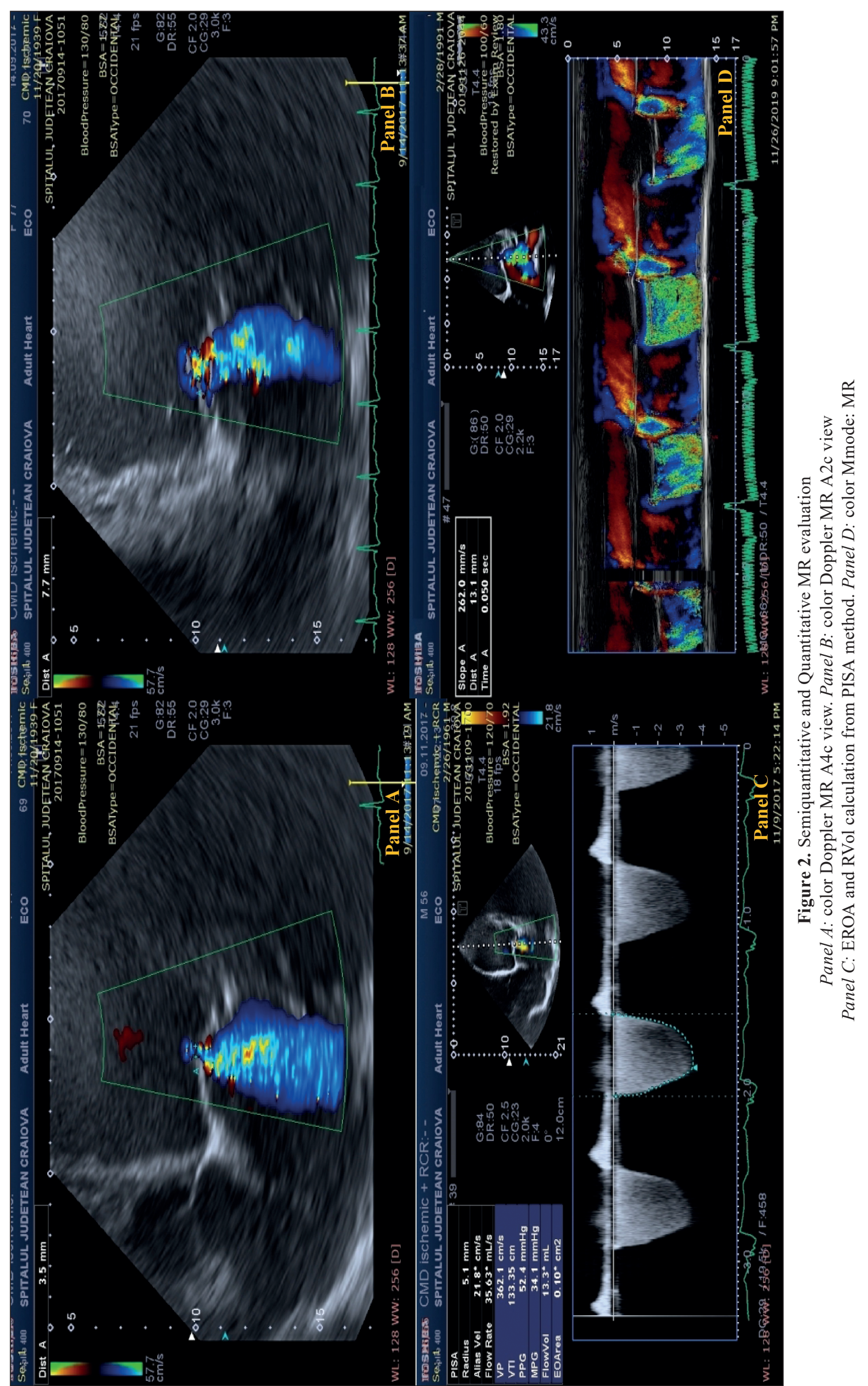




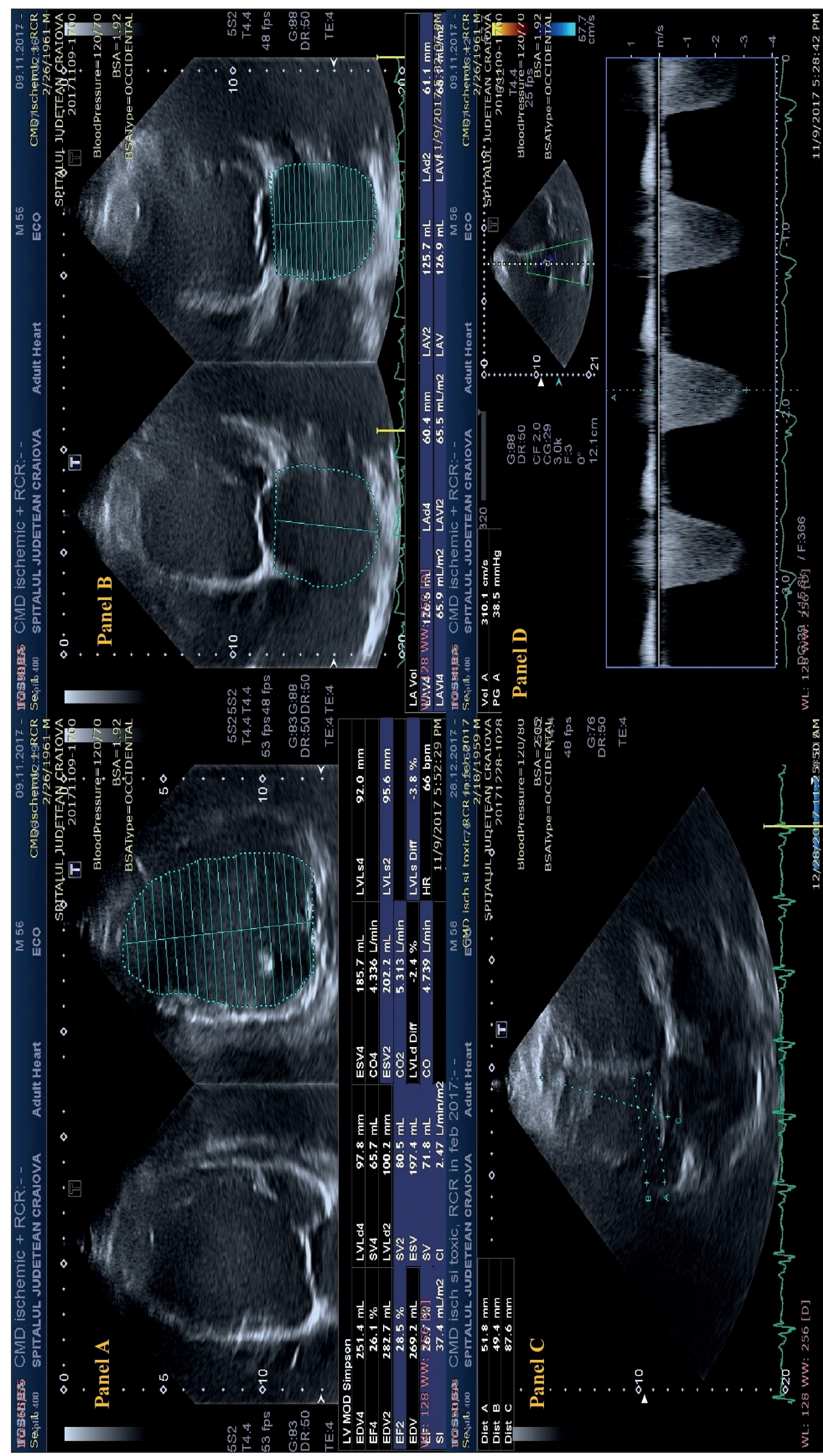

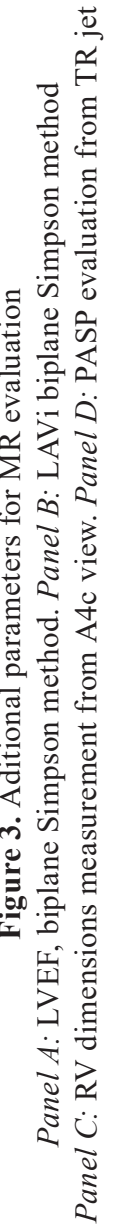




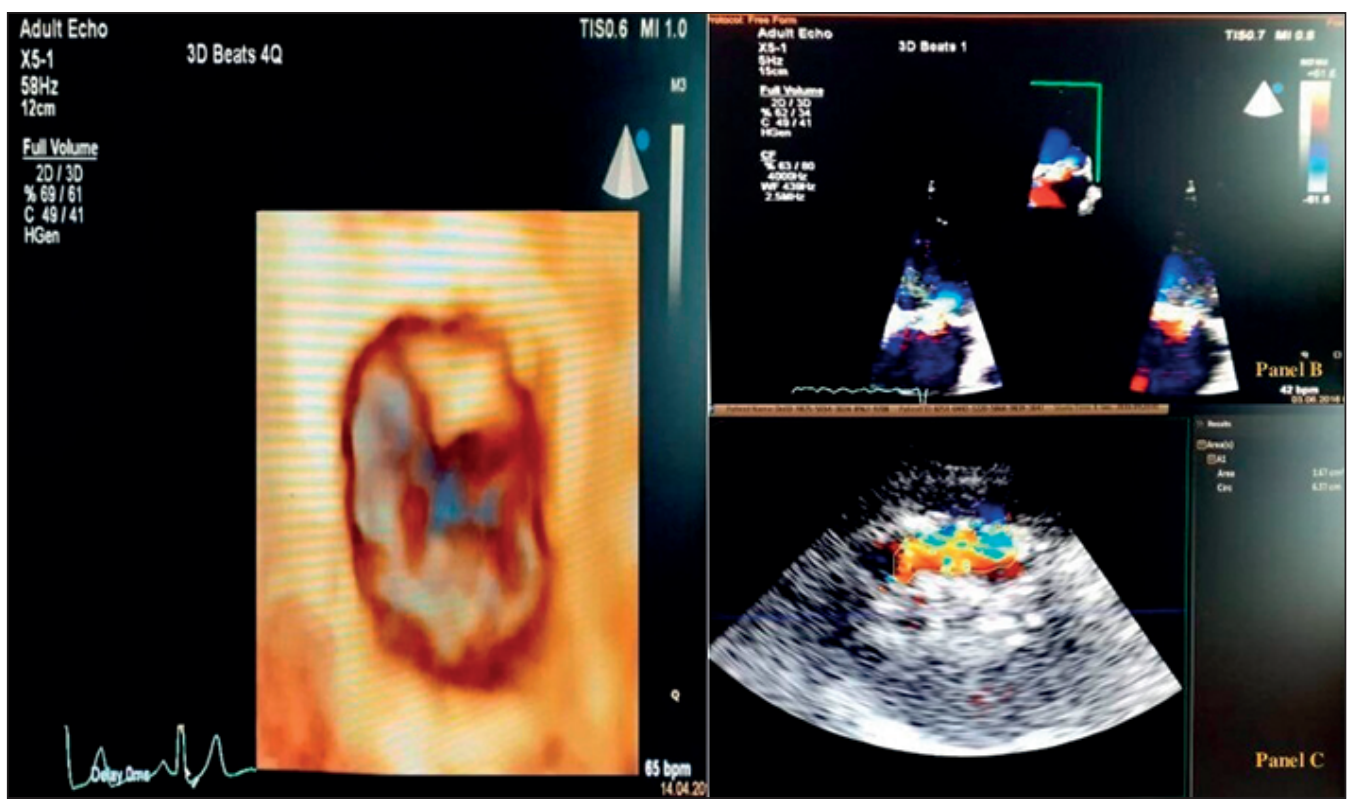

Figure 4. 3D echocardiography

Panel A: MV „en face view“. Panel B: color VC evaluation, in A4c view, in A2c view, and along the commissural line „en face“ view. Panel C: color VC measurement from „en face“ view

originating from the medial and lateral sides of the coaptation line. When the shape of the flow convergence zone is not a hemisphere, and the ratio of long-axis length to short-axis length of the regurgitant orifice is $>1.5$, the PISA method may underestimate the degree of functional mitral regurgitation. It is not valid for multiple jets.?

PISA method is highly recommended by the guidelines to evaluate the severity of regurgitation whenever feasible.?

In conclusion, FMR severity may be overestimated if it is determined based on the color jet size or underestimated when is assessed using other traditional measures of mitral regurgitation such as PISA and VC width.?

A complete 2D transthoracic echocardiography report of FMR evaluation after CRT must provide supplementary information about:

- Ventricular (Figure 3, Panel A) and atrial size (Figure 3, Panel B) (assessed by the Simpson method). Dilatation is sensitive for chronic severe regurgitation, but it is non-specific, because it could be observed in other conditions?

- The right atrium and right ventricle dimensions and functions ${ }^{6}$ (Figure 3, Panel C).

- Estimated pulmonary artery systolic pressure, considering that patient has an implanted device, which usually interferes with tricuspid valve closure $^{6}$ (Figure 3, Panel D).

3D echocardiography adds information about severity, because orifice geometry in functional MR is noncircular or there are multiple jets. ${ }^{3}$ This technique overcomes the limitations of 2D imaging and it was superior to describe mitral valve pathology. 3D echocardiography enables visualization from the left ventricle and left atrium perspectives. The "front" view of the valve from the left atrium perspective is identical to the surgical view in the operating room (Figure 4, Panel A).

For TTE 3D evaluation of the mitral valve, guidelines recommend narrow angle and zoomed acquisitions from parasternal long-axis view and apical four-chamber view. ${ }^{8}$ A zoomed acquisition has the highest temporal and spatial resolution and is recommended for a detailed evaluation of leaflets anatomy and motion. A full-volume acquisition is required when the entire mitral valve apparatus needs to be assessed. 8

The posterior mitral valve leaflet is best visualized from the parasternal window, while the anterior leaflet can be well seen from both apical and parasternal windows. Sub valvular apparatus can be appreciated from left ventricle long-axis cut planes. The mitral annulus is best appreciated from the 3D surgical view of the mitral valve. ${ }^{3}$

For mitral valve competence during systole, normal motion and contraction of both ventricles and mitral annulus are required. A change in left ventricle geometry that affects papillary muscles position results in poor leaflet coaptation. Dynamic 3D rendering of the mitral valve can differentiate between normal leaflet mobility and tethered leaflets due to regional wall motion abnormalities or global left ventricular enlargement. ${ }^{3}$ 
Table 1: Difference in Means values of FMR in CARE-HF trial at 3 months and 18 months

\begin{tabular}{ccccc}
\hline FMR $^{*}$ & Difference in Means at 3 Mo $(95 \% \mathrm{CI})$ & $P$ & Difference in Means at 18 Mo (95\% CI) & $p$ \\
\hline$-0.051(-0.073$ to -0.028$)$ & $<0.001$ & $-0.042(-0.070$ to -0.014$)$ & 0.003 \\
\hline
\end{tabular}

*The area was calculated as the area of the color-flow Doppler regurgitant jet divided by the area of the left atrium in systole, in square centimeters.

FMR: mitral regurgitation, $\mathrm{CI}$ : confidence interval

Table 2: FMR evaluation in MIRACLE trial

\begin{tabular}{|c|c|c|c|c|c|c|}
\hline \multirow{3}{*}{ Parameter } & \multicolumn{6}{|c|}{ FMR } \\
\hline & \multicolumn{3}{|c|}{ Control Group ( $n=151)$} & \multicolumn{3}{|c|}{ CRT Group $(n=172)$} \\
\hline & Baseline & 3 Months & 6 Months & Baseline & 3 Months & 6 Months \\
\hline average FMR jet area, $\left(\mathrm{cm}^{2}\right)$ & $7.30-5.12$ & $0.1(-0.7,0.7)$ & $-0.5(-1.1,0.2)$ & $7.31-6.14$ & $-2.1(-3.0,-1.2)$ & $-2.5(-3.3,-1.7)$ \\
\hline
\end{tabular}

FMR: functional Mitral Regurgitation

At this moment, clinical validation and application evaluation of the mitral apparatus using 3D echocardiography is useful for:

- defining the extent and location of the pathology;

- determining the mechanism and severity of valvular dysfunction;

- communicating the results to the interventional cardiologist or cardiac surgeon if an intervention is required. ${ }^{8}$

Delineation of the effective regurgitant orifice area and the $\mathrm{VC}$ are two major strengths of 3D echocardiography. ${ }^{8}$

3D derived $\mathrm{VC}$ area has been shown to correlate more closely with Doppler-derived effective regurgitant orifice area than 2D VC diameter. The VC area is narrow in the four-chamber view, broad in the two-chamber view, and asymmetric along the commissural line in a "front" view (Figure 4, Panel B). The planar area measured from "front" view is the parameter which corresponds directly to the effective regurgitant orifice area, irrespective of the orifice shape or number of jets ${ }^{8}$ (Figure 4, Panel C). With 3D echocardiography and color flow imaging, it is also possible to quantitate mitral regurgitant jet volumes. Guidelines recommend 3D echocardiography as a valuable tool in mitral regurgitation assessment, particularly in those patients with regurgitation underestimated by 2D imaging methods or with complex anatomy. ${ }^{6}$ 3D assessments of mitral valve pathology should be incorporated into routine clinical practice as they provide the best physiologic and morphologic information regarding the mitral valve. ${ }^{6}$

However, both 3D and 2D color Doppler flow tend to overestimate the jet area. ${ }^{6}$

\section{Methods used in clinical trials evaluating FMR IN CRT PATIENTS}

Only three trials in patients with CRT have included mitral regurgitation in their evaluation outcomes: CARE-HF, MIRACLE, and MUSTIC, but these studies were pointed to heart failure and did not evaluate specifically FMR. The evaluation had many limitations regarding the methods of quantification. The Cardiac Resynchronization - Heart Failure (CARE-HF) trial used for FMR evaluation of the jet area and the mitral regurgitation jet area/left atrium area ratio. The jet area was used in The Multicenter InSync Randomized Clinical Evaluation (MIRACLE) trial and Multisite Stimulation in Cardiomyopathies (MUSTIC) trial.

CARE-HF was a multicenter, international, randomized trial comparing the effect of standard pharmacologic therapy alone with that of the combination of standard therapy and cardiac resynchronization (without a defibrillator) on the risk of complications and death in patients with left ventricular systolic dysfunction, cardiac dyssynchrony, and NYHA class III or IV heart failure. 409 CRT-on patients were enrolled and followed for a mean of 29.4 months. At both 3 months and 18 months, the area of mitral regurgitation was significantly smaller in the CRT group than in the medical therapy group (Table 1). Favorable results were also obtained on cardiac dyssynchrony and LV remodeling. The benefits were similar among patients with or without ischemic heart disease.

MIRACLE was a prospective, double-blind, randomized, controlled trial, in which 373 patients on optimal medical heart failure treatment regimen were randomized to CRT or no CRT. 172 patients were CRT-on. In this group, the severity of mitral regurgitation decreased significantly at 3 months and at 6 months (Table 2). Patients with moderate-to-severe heart failure who received CRT also presented reverse left ventricular remodeling, improved systolic and diastolic function. Results were better in patients with a nonischemic versus ischemic cause of heart failure. ${ }^{10}$

MUSTIC was a randomized controlled study that intended to evaluate the effects of biventricular 
Table 3: FMR evaluation in MUSTIC trial

\begin{tabular}{|c|c|c|c|c|}
\hline \multicolumn{5}{|c|}{$F M R$} \\
\hline & Randomization & 6 Months & 9 Months & 12 Months \\
\hline & \multicolumn{4}{|c|}{ Sinus rhythm } \\
\hline \multirow{7}{*}{ FMR area $\left(\mathrm{cm}^{2}\right)$} & $7.4-6.8(n=44)$ & $5.6-8.3(n=44)$ & & \\
\hline & $8.0-7.8(n=39)$ & & $4.9-4.6(n=39)$ & \\
\hline & $7.8-7.8(n=39)$ & & & $4.3-4.0(n=39)$ \\
\hline & \multicolumn{4}{|c|}{$\mathrm{AF}$} \\
\hline & \multicolumn{3}{|c|}{ NA } & \\
\hline & $10.2-13.7(n=27)$ & & $6.4-6.2(n=27)$ & \\
\hline & $10.8-13.7(n=26)$ & & & $5.4-3.9(n=26)$ \\
\hline
\end{tabular}

FMR: functional Mitral Regurgitation

pacing in patients with NYHA class III heart failure and intraventricular conduction delay. 131 patients were included (34 CRT-on) and were followed up longitudinally at 9 and 12 months. Compared with baseline, the mitral regurgitation decreased by $45 \%$ sinus rhythm patients and $50 \%$ atrial fibrillation patients. For the sinus rhythm group, the mitral regurgitation had decreased markedly at 6 months, at 9 months, and at 12 months. For the atrial fibrillation group, the mitral regurgitation also decreased at 9 months and at 12 months compared with baseline values. ${ }^{11}$ (Table 3)

In CARE-HF study, the mitral regurgitation jet area decreased by $34 \%(p<0.05)$ at 3 months and it was the strongest predictor of mortality. In MIRACLE study, the mitral regurgitation was reduced by $29 \%$ ( $p<0.05$ ) at 3 months. In MUSTIC trial, the mitral regurgitation area jet decreased by $23 \%(\mathrm{p}<0.05)$ after 6 months. ${ }^{11}$ These results highlight the prognostic importance of FMR in patients who were responders to CRT.

\section{Methods used for FMR evaluation short-term after CRT}

Several echocardiographic parameters were used in different small studies for evaluation of early response of FMR at CRT.

The acute response was demonstrated in the study of Breithardt et al, in which mitral regurgitation was evaluated by regurgitant volume and effective regurgitant orifice area, using the PISA method, in the first week after CRT. Reduction in mitral regurgitation was directly related to the increase in papillary muscle contraction measured by longitudinal strain. ${ }^{12}$ Kazanski et al used for acute MR reduction evaluation after CRT the regurgitation volume obtained with the volumetric method. This was associated with improved coordination of the left ventricular segments containing papillary muscles. ${ }^{13}$

Presystolic component disappears early, with the pacemaker activation; CW Doppler signal intensity is also reduced after CRT, suggesting an improvement of the regurgitation severity. ${ }^{4}$ Nof $\mathrm{E}$ et al demonstrated that diastolic component was eliminated in all patients early after CRT. This study revealed that mitral regurgitation tethering was a constitutive element of the mechanism of diastolic mitral regurgitation in patients with left ventricular dysfunction. ${ }^{14}$

Local myocardial viability was demonstrated to be an important predictor of FMR response to CRT. ${ }^{4}$ Goland et al confirmed that the presence of significant time-to-peak delay between inferior and anterior left ventricular segments, preserved strain of posterior wall, and mitral regurgitation jet area/ left atrium area ratio $<40 \%$ were factors associated with significant mitral regurgitation reduction after CRT implantation. ${ }^{15}$ In the study of Onishi et al, 277 patients were evaluated using for mitral regurgitation quantification a multiparametric approach: $\mathrm{VC}$ width and mitral regurgitation jet area/left atrium area ratio. Radial dyssynchrony was measured by speckle tracking radial strain from the mid-left ventricular short-axis view. Mitral regurgitation reduction after CRT and long-term survival were correlated with baseline radial dyssynchrony, excessive left ventricular dilatation, and absence of the scar at the papillary muscles insertion site. ${ }^{16}$ Brezinska et al also assessed the predictive role of the myocardial contractile reserve for FMR improvement after CRT. The severity of FMR was estimated semi-quantitatively using $\mathrm{VC}$ and mitral regurgitation jet area/left atrium area ratio. Contractile reserve preserved in more than three segments was a predictor for short-term FMR improvement and this was associated with a higher incidence of the early left ventricular reverse remodeling, in the six weeks after CRT. ${ }^{17}$

\section{Methods used for FMR evaluation long-term after CRT implantation}

Many studies showed a reduction in FMR severity with the restoration of synchronous ventricular 
contraction and left ventricular remodeling ${ }^{18}$. Porciani et al evaluated FMR by mitral regurgitation jet area/left atrium area ratio and dyssynchrony by the standard deviation of the time to the systolic peak velocity at 6-basal and mid-left ventricular segments measured by tissue Doppler echocardiography. FMR improvement after CRT has been related to left ventricular remodeling reversal and contractility enhancement. ${ }^{19} 302$ patients were followed up at 6 months after CRT by Ypenburg et al, mitral regurgitation was graded semi quantitatively using the mitral regurgitation jet area/left atrium area ratio. The reduction was more pronounced in patients with more left ventricular reverse remodeling. ${ }^{20}$ In the study of Cabrera-Bueno et al, for mitral regurgitation grade assessment, the effective orifice regurgitant area was used. This became non-significant in more than one third of these cases after implant and the persistence was confirmed as a bad prognostic factor, associated with less left ventricular reverse remodeling after 12 months of follow-up. ${ }^{21}$ Di Biase et al followed up 794 CRT patients, assessing mitral regurgitation severity and left ventricular reverse remodeling at baseline, 3 , and 12 months after implantation. In this study, effective regurgitant orifice area, $\mathrm{VC}$, jet area, and multiparametric grading were used. Mitral regurgitation grades 3 and 4 at baseline and improvement in mitral regurgitation $>1$ grade at the 3-month follow-up predicted response to CRT. Based on the results of this study, Di Biase et al proposed an algorithm of following up the patients at 3 months after CRT, because at this time some patients could have the mitral regurgitation improved by CRT alone. If no improvement occurs, it is unlikely that a further improvement of mitral regurgitation could be obtained. ${ }^{22}$

In Van Bommel et al study, survival at 6 months was superior in mitral regurgitation improvers compared with MR non-improvers. Parameters used for mitral regurgitation evaluation were: $\mathrm{VC}$ and effective regurgitation orifice area. Mitral regurgitation reduction independently of the end-systolic volume response was predictive of favorable CRT response. ${ }^{23}$

Verhaert et al demonstrated that early reversal of FMR was associated with reverse cardiac remodeling and improved outcomes. 266 CRT patients were followed for 3 months. The severity of mitral regurgitation was quantified by $\mathrm{VC}$, mitral regurgitation jet area/left atrium area ratio, and effective regurgitation orifice area. Patients with moderately-severe to severe mitral regurgitation before CRT experienced relatively more reverse remodeling than patients with lesser degrees of mitral regurgitation. ${ }^{24}$

In the study of Mihos et al, 71 patients with mild or greater secondary mitral regurgitation undergoing CRT were mid-term followed up by analysis of the mitral valve apparatus geometry and VC measurement. The 2D echocardiographic parameters evaluated were: mitral valve tenting height, mitral valve tenting area, anterior and posterior leaflet tethering angles, coaptation length, interpapillary muscle distance, posterior ventricular sulcus-anterolateral papillary muscle angle, mitral annular diameter. They found CRT favorable effects on mitral valve and left ventricle geometry and decrease of the prevalence of moderate-to-severe or severe FMR. ${ }^{25}$

Der Bijl et al investigated the prevalence, evolution, and impact on mortality of FMR before and after CRT in patients with heart failure. A total of 1313 patients were evaluated. The severity of FMR was evaluated using a multiparametric approach, including qualitative, semi-quantitative and quantitative mitral regurgitation indices. Moderate to severe FMR at baseline which remains unchanged at 6 months after CRT implantation was strongly associated with long-term mortality. The FMR changes were related to 6 months response to CRT. ${ }^{26}$

Solis et al evaluated FMR before and 6 months after CRT using 3D echocardiography parameters. The principal objective of this study was to examine the effects of CRT on the mitral valve. Regurgitation volume, closing forces on mitral valve, including $\mathrm{dP} / \mathrm{dt}$, and closing pressure ratio were measured. Dyssynchrony was evaluated by tissue Doppler. Mitral valve annular area and contraction, leaflet closing area, and tenting volume were measured by $3 \mathrm{D}$ echocardiography. Reduction in mitral regurgitation after CRT was associated with favorable changes in mitral valve geometry and closing forces. Beneficial left ventricular geometric and volumetric changes after CRT from reverse remodeling affected mitral valve annular area, leaflet closing area, and tenting volume. The study concluded that an excessive left ventricular dilatation may be a marker for lack of improvement in mitral regurgitation or a new development and nonresponse to CRT and may be associated with the development of new mitral regurgitation after CRT. ${ }^{27}$

In a review of Asgar et al, regarding the secondary mitral regurgitation in heart failure, they also pointed out that accurate imaging assessment of secondary mitral regurgitation may be challenging. No single parameter is sufficient to quantify the degree of mitral regurgitation, and multimodality assessment using both 2D and 3D echocardiography is optimal. ${ }^{28}$

\section{Conclusions}

Mitral regurgitation is a frequent complication in patients with CRT. Its development is the result of the imbalance between closure forces and tethering forces secondary to a disruption in the normal 
left ventricular geometry and a dyssynchronous left ventricular contraction.

It is important to use an integrated approach when assessing mitral regurgitation in patients after CRT implantation, usually after 3 months.

CRT is associated with a reduction in FMR, and this decrease in FMR probably influences the favorable effects of CRT.

The echocardiographic assessment of FMR includes integration of data from 2D/3D imaging, as well as Doppler measures of regurgitation severity. For quantifying the regurgitation degree, the VC width and the parameters derived from PISA method: effective regurgitant orifice area and regurgitation volume are the most useful. There is also important to provide information about the left ventricle and right ventricle dimensions and LVEF, as well as the left atrium, right atrium dimensions and the pulmonary systolic arterial pressure.

\section{Author Contributions:}

D.M.T. conceived the original draft preparation and was responsible for conception and design of the review, inclusion of the articles/published data and interpret them in the review. C.B. was responsible for the collection and assembly of the articles/published data. R.M. contributed to the conception and design of the review. All the authors were responsible for data acquisition. All the authors contributed to the critical revision of the manuscript for valuable intellectual content. All the authors have read and agreed with the final version of the manuscript.

\section{Compliance with Ethics Requirements:}

"The authors declare no conflict of interest regarding this article"

„The authors declare that all the procedures and experiments of this study respect the ethical standards in the Helsinki Declaration of 1975, as revised in 2008(5), as well as the national law. Informed consent was obtained from all the patients included in the study"

"No funding for this study"

\section{Acknowledgements:}

None

\section{References}

1. Ponikowski P, Voors AA, Anker SD, et al. 2016 ESC Guidelines for the diagnosis and treatment of acute and chronic heart failure. The Task Force for the diagnosis and treatment of acute and chronic heart failure of the European Society of Cardiology (ESC) Developed with the special contribution of the Heart Failure Association (HFA) of the ESC. European Heart Journal. 2016; 37:2129-2200.
2. Brignole M, Auricchio A, Baron-Esquivias, et al. 2013 ESC Guidelines on cardiac pacing and cardiac resynchronization therapy. The Task Force on cardiac pacing and resynchronization therapy of the European Society of Cardiology (ESC). Developed in collaboration with the European Heart Rhythm Association (EHRA). European Heart Journal. 2013; 34:2281-2329.

3. Galiuto L, Badano L, Fox K, et al. The EAE Textbook of Echocardiography. Oxford University Press New York. 2011;238-244.

4. Spartera M, Galderisi M, Mele D, et al. On behalf of the Echocardiographic Study Group of the Italian Society of Cardiology (SIC). Role of cardiac dyssynchrony and resynchronization therapy in functional mitral regurgitation. European Heart Journal - Cardiovascular Imaging. 2016; 17:271-280.

5. Donal E, De Place C, Kervio G, et al. Mitral regurgitation in dilated cardiomyopathy: value of both regional left ventricular contractility and dyssynchrony. European Journal of Echocardiography. 2009; 10:133-138.

6. Baumgartner H, Falk V, Bax JJ, et al. 2017 ESC/EACTS Guidelines for the management of valvular heart disease. The Task Force for the management of valvular heart disease of the European Society of Cardiology (ESC) and the European Association for Cardio-Thoracic Surgery (EACTS). European Heart Journal. 2017; 38:2739-2791.

7. Lancellotti P, Tribouilloy C, Hagendorff A, et al. Recommendations for the echocardiographic assessment of native valvular regurgitation: an executive summary from the European Association of Cardiovascular Imaging. European Heart Journal - Cardiovascular Imaging. 2013; 14:611-644.

8. Lang MR, Badano LP, Tsang W, et al. EAE/ASE Recommendations for image acquisition and display using three-dimensional echocardiography. European Heart Journal - Cardiovascular imaging. 2012; 13:3-46.

9. Cleland JGF, Daubert J-C, Erdmann E, et al. For the Cardiac Resynchronization - Heart Failure (CARE-HF) Study Investigators. The effect of cardiac resynchronization on morbidity and mortality in heart failure. New England Journal of Medicine. 2005; 15:1539-1549.

10. St John Sutton MG, Plappert T, Abraham WT, et al. For the Multicenter InSync Randomized Clinical Evaluation (MIRACLE) Study Group. Effect of cardiac resynchronization therapy on left ventricular size and function in chronic heart failure. Circulation. 2003; 24:1985-1990.

11. Linde C, Leclercq C, Rex S, et al. Long-term benefits of biventricular pacing in congestive heart failure: Results from the Multisite STimulation In Cardiomyopathy (MUSTIC) Study. Journal of the American College of Cardiology. 2002; 40:111-118.

12. Breithardt OA, Sinha AM, Schwammenthal E, et al. Acute effects of cardiac resynchronization therapy on functional mitral regurgitation in advanced systolic heart failure. Journal of the American College of Cardiology. 2003; 41:765-770.

13. Kanzaki H, Bazaz R, Schwartzman D, Dohi K, Sade LE, Gorcsan J. A mechanism for immediate reduction in mitral regurgitation after cardiac resynchronization therapy insights from mechanical activation strain mapping. Journal of the American College of Cardiology. 2004; 8:1619-1625.

14. Nof E, Glikson M, Bar-Lev D, et al. Mechanism of diastolic mitral regurgitation in candidates for cardiac resynchronization therapy. Journal of the American College of Cardiology. 2006; 97:1611-1614. 
15. Goland S, Rafique AM, Mirocha J, Siegel RJ, Naqvi TZ. Reduction in mitral regurgitation in patients undergoing cardiac resynchronization treatment: assessment of predictors by two-dimensional radial strain echocardiography. Echocardiography. 2009; 26: 420-430.

16. Onishi T, Onishi T, Marek JJ, et al. Mechanistic features associated with improvement in mmitral regurgitation after cardiac resynchronization therapy and their relation to long-term patient outcome. Circulation - Heart Failure. 2013; 6:685-693

17. Brzezińska B, Łoboz-Grudzień K, Wita K, et al. Predictors of functional mitral regurgitation improvement during a short-term follow-up after cardiac resynchronisation therapy. Kardiologia Polska. 2016; 74:665-673.

18. Moisi MI, Rus M, Bungau S, et al. Acute coronary syndromes in chronic kidney disease: clinical and therapeutic characteristics. Medicina. 2020;56:118.

19. Porciani MC, Macioce R, Demarchi G, et al. Effects of cardiac resynchronization therapy on the mechanisms underlying functional mitral regurgitation in congestive heart failure. European Journal of Echocardiography. 2006; 7:31-39.

20. Ypenburg C, van Bomme RJ, Borleffs JW, et al. Long-term prognosis after cardiac resynchronization therapy is related to the extent of left ventricular reverse remodeling at midterm follow-up. Journal of the American College of Cardiology. 2009; 53:483-490

21. Cabrera-Bueno F, Molina-Mora MJ, Alzueta J, et al. Persistence of secondary mitral regurgitation and response to cardiac resynchronization therapy. European Journal of Echocardiography. 2010; 11:131-137.

22. Di Biase L, Auricchio, Mohanty P, et al. Impact of cardiac resynchronization therapy on the severity of mitral regurgitation. Europace. 2011; 13:829-838.

23. Van Bommel RJ, Marsan NA, Delgado V, et al. Cardiac resynchronization therapy as a therapeutic option in patients with moderate-severe functional mitral regurgitation and high operative risk. Circulation. 2011; 124:912-919.

24. Verhaert D, Popovic ZB, De S, et al. Impact of mitral regurgitation on reverse remodeling and outcome in patients undergoing cardiac resynchronization therapy. Circulation Cardiovascular Imaging. 2012; 5:21-26.

25. Mihos CG, Evin Yucel E, Capoulade R, et al. Impact of cardiac resynchronization therapy on mitral valve apparatus geometry and clinical outcomes in patients with secondary mitral regurgitation. Echocardiography.2017; 34:1561-1567.

26. Van der Bijl P, Khidir M, Marsan NA, et al. Effect of functional mitral regurgitation on outcome in patients receiving cardiac resynchronization therapy for heart failure. Journal of the American College of Cardiology. 2019; 123:75-83.

27. Solis J, McCarty D, Levine RA, et al. Mechanism of decrease in mitral regurgitation after cardiac resynchronization therapy optimization of the force-balance relationship. Circulation - Cardiovascular Imaging. 2009; 2:444-450.

28. Asgar AW, Mack MJ, Stone GW, et al. Secondary mitral regurgitation in heart failure pathophysiology, prognosis, and therapeutic considerations. Journal of the American College of Cardiology. 2015; 65:1231-1248. 